\title{
A free software package for a human online- conditioned suppression preparation
}

\author{
MathiJs Franssen and Jeroen Clarysse \\ University of Leuven, Leuven, Belgium \\ TOM BECKERS \\ University of Amsterdam, Amsterdam, The Netherlands \\ and University of Leuven, Leuven, Belgium \\ AND \\ Priya R. VAN VOOREN AND Frank BaEYens \\ University of Leuven, Leuven, Belgium
}

\begin{abstract}
MartiansV2 is both a language syntax in which experiments can be written and an implementation of this syntax in a runtime application that, when fed a valid experiment text file, will execute the given experiment. It is based on the original Martians preparation, which has proven a valuable tool for assessing human onlineconditioned suppression performance through research on a wide array of learning phenomena. This article can be read as a manual, both for using the Martians paradigm in general and for getting started with MartiansV2.
\end{abstract}

The Martians preparation was first used by Arcediano, Ortega, and Matute (1996). Their main reason for introducing the paradigm was the search for a "human" preparation similar to those used in animal conditioning research. More specifically, they wanted to obtain a human counterpart for animal conditioned suppression preparations. In a typical experiment assessing onlineconditioned suppression in rats, subjects can obtain food or water by barpressing. During reinforced trials, a conditioned stimulus (CS; e.g., a tone or light) is followed by an aversive unconditioned stimulus (US; e.g., footshock). The latter unconditionally suppresses the subjects' barpressing behavior. While learning the CS-US association, the subjects will show progressively more conditioned responding on CS presentations (i.e., they will come to suppress barpressing on CS presentation).

Because of ethical and moral limitations (regarding the use of shocks or other aversive USs), causal judgment tasks (see, e.g., Beckers, De Houwer, Pineño, \& Miller, 2005) are often used with human subjects, as an alternative to conditioned suppression procedures. However, the responses obtained from subjects in such studies are of an explicit and verbal nature. Acknowledging the usefulness of causal judgment tasks and their analogies to animal conditioning procedures, Arcediano et al. (1996) nonetheless argued that a preparation that measures behavior instead of verbal judgments would be considerably more similar to procedures used in animal research. They therefore decided to implement instructed instead of aver- sive USs in order to make a human online-conditioned suppression procedure feasible. Also, we would like to stress that, during the Martians task, (not) responding has to be decided under time pressure. This property is often neglected but may influence the outcome, and is definitely different in standard causal judgment tasks.

\section{THE MARTIANS PREPARATION}

In the Martians task, human subjects are instructed to prevent as many Martians as possible from invading the Earth by shooting them - that is, continuously pressing the space bar (operant responding). However, the Martians will sometimes activate an antilaser shield. Whenever this shield is activated, shots against it ricochet back onto the shooter, leaving the subject helpless while massive numbers of Martians unavoidably invade the Earth (instructed US). Different predictors can come up during the task (CS). Good predictors are followed by activation of the antilaser shield (CS+), whereas bad predictors are not (CS-). It is up to the subjects to learn to discriminate bad from good predictors; they should stop barpressing in the presence of a good predictor and thus avoid provoking invasions. Since the predictors can consist of several stimuli (e.g., $\mathrm{X}$ and A), presented simultaneously or sequentially, more complex patterns, such as sequential feature positive discriminations $\left(\mathrm{X} \rightarrow \mathrm{A}^{+} / \mathrm{A}^{-}\right)$, can easily be implemented. For a conceptual discussion on operant versus Pavlovian conditioning interpretations of the Mar-

M. Franssen, mathijs.franssen@psy.kuleuven.be 
tians preparation, see Baeyens, Vansteenwegen, Hermans, Vervliet, and Eelen (2001).

Explaining the aim of the game to subjects is usually done by including two pretraining phases, a barpressing phase and a US-only phase.

\section{Barpressing Pretraining}

The purpose of the barpressing phase is to teach subjects to emit a regular pattern of operant responding - that is, to barpress consistently using the space bar of the keyboard. During this phase, no CSs or USs are presented. The screen displays incoming Martians trying to invade the Earth, and the task of the subjects is to prevent them from doing so by firing a laser gun at them. At each hit, an explosion, not a Martian, is displayed. Subjects receive onscreen instructions (included in the example in the System Requirements and Downloads section), after which the experimenter allows them to practice barpressing (shooting Martians) for $25 \mathrm{sec}$ (100 Martians and/or explosions, at a rate of $4 / \mathrm{sec}$ ). If the subject does not instantly grasp the instructions, the experimenter demonstrates by barpressing for a few seconds. Also, the experimenter points out that it is not advisable to barpress too fast, since there is only one shot available per Martian. At the end of the phase, the percentage of hits is displayed on the screen as feedback.

\section{US-Only Pretraining}

Before starting the US-only phase, the subject receives further on-screen instructions. The instructional US is introduced through description and demonstration of what happens if subjects keep shooting during presentations of the antilaser shield. A US consists of a 0.5 -sec simultaneous presentation of a screen flashing intermittently white, against an associated sound background. During the US, the Martians keep appearing on the screen in the same way as during the intertrial interval (ITI; 4/sec). If barpressing is stopped in time, nothing new happens. If barpressing is continued, a screen flashing white and a loud metal sound create the equivalent of a shock, interrupting the operant baseline task: This is called an invasion. An invasion lasts for $5 \mathrm{sec}$, during which the background keeps flashing (10 flashes/sec), a new sound pattern is played, and Martians invade the screen at a rate of $10 / \mathrm{sec}$. Moreover, barpressing is ineffective during an invasion (no explosions appear contingent on barpressing). In total, four USs are presented (average ITI of $7.5 \mathrm{sec}$; minimum 5, maximum 10). None of them is signaled by a preceding stimulus. The first and second US presentations are used by the experimenter to demonstrate the instructions. During the first presentation, the experimenter refrains from barpressing to ensure that the subject fully understands which events represent activation of the antilaser shield. During the second presentation, the experimenter continues barpressing to show the subject what an invasion looks like. Thereafter, the subject takes over. Afterward, feedback is printed on the screen, and the experimenter indicates how provoking an invasion has a devastating effect on the percentage of hits.
The experimenter introduces the subsequent acquisition phase by telling the subjects that, as they probably understand, it would be very beneficial to have a means of predicting the activation of the antilaser shield. A final set of instructions is displayed, explaining that different indicators may be introduced. It is up to the subject to learn to discriminate the rather good ( $\mathrm{CS}+$ ) from the rather bad $(\mathrm{CS}-)$ shield-activation predictors, thereby learning when to stop $(\mathrm{CS}+)$ or to continue barpressing $(\mathrm{CS}-)$.

\section{MARTIANSV2}

As stated by Matute, Vadillo, and Bárcena (2007, p. 690), "In principle, any experiment that could use suppression ratio with animals as the index that the association has been acquired can also be conducted with humans using this task." In other words, applications for the Martians preparation can be very diverse, containing at the very least (conceptually) all experiments with animals that measure suppression ratios. In fact, the preparation has already proven its worth for investigating a diverse array of learning phenomena. So far, Martians has been successfully used to investigate blocking (Arcediano, Matute, \& Miller, 1997) and other forms of cue competition (Matute \& Pineño, 1998), simultaneous and sequential feature positive discriminations (Baeyens et al., 2001), simultaneous and sequential feature negative discriminations (Baeyens et al., 2004), extinction, renewal, and reinstatement of modulation (Baeyens et al., 2005; Fonteyne \& Baeyens, 2009; Franssen, Gillard, Dirikx, van Vooren, \& Baeyens, 2009), contextual modulation and extinction (Havermans, Keuker, Lataster, \& Jansen, 2005), the influence of unpredictability-induced context conditioning on subsequent learning to a discrete cue (Meulders, Vervliet, Vansteenwegen, Hermans, \& Baeyens, 2009), cue competition effects after elementary training (Lipp \& Dal Santo, 2002), and consolidation of the CS-US association through mental rehearsal (Joos, Vansteenwegen, \& Hermans, 2008). Outside learning psychology, the preparation could also be used, for example, to investigate hypotheses concerning avoidance mechanisms, such as a simple game-oriented filler task; or it could simply be used as a means of presenting electrical shocks.

To provide this amount of flexibility, almost all experimental parameters are adjustable (e.g., background, CS duration, CS modality, type of Martians, duration of invasion, etc.) and one can measure the main dependent variable, counts of barpresses, at any moment. Also, MartiansV2 allows the administration of electric stimuli (but not necessarily as USs). Although these examples clearly indicate that the preparation is suitable for investigating complex learning phenomena, the goal of MartiansV2 is to render the paradigm usable for professional researchers, as well as for undergraduate and graduate students. Through years of intensively and extensively applying the preparation, the program used for creating the actual experiments underwent several changes. The original preparation developed by Arcediano et al. (1996) had already been implemented into a flexible Windows 95 environment by Baeyens and 
Clarysse (1998) using Microsoft Visual C++ 5.0. For the subjects, the main implication was that all visual and sound effects were somewhat more attractive and sophisticated than were those produced by the original Arcediano et al. (1996) program. Also, researchers could easily use the programs' graphical user interface (GUI) to create new experiments. The new MartiansV2 (Baeyens, Clarysse, Franssen, \& Hermans, 2008) is designed to further improve flexibility for the experimenter in both present and future endeavors. In order to guarantee this flexibility (including adding new features easily, copy-paste-wise recycling of older code, cross-platform usage, etc.), we abandoned the GUI. MartiansV2 experiments consist of text files - more precisely, of code written in the MartiansV2 syntax. They can be opened by any plain text editor ${ }^{1}$ on both Mac- or Windows-based platforms, and can largely be read without any knowledge of the code. MartiansV2 is a command-line application, called from a command prompt (a DOS box in Windows or the Terminal on Mac) when the location and name of the program are typed in, followed by the location and name of a valid experiment file. However, to make things easier, Windows users can also double-click the application, which will then launch itself in a new DOS box. Mac users can launch the experiment directly from Textwrangler, a free general-purpose text editor.

\section{SYNTAX}

So that it would be usable by anyone interested in conducting learning experiments, the MartiansV2 syntax has been kept very comprehensible. The basics of writing code will now be explained, using a simple differential learning task $\left(\mathrm{A}^{+} / \mathrm{B}^{-}\right)$as an example. Our goal here is not to provide a comprehensive manual, but to present an example that illustrates the ease with which experiments can be programmed. Hereafter, MartiansV2 code will be printed in a different font (e.g., smarter invasion). Text references to code will be placed between quotation marks (e.g., "martians version"). The entire example, both code as well as instructions and stimuli, can be downloaded from our site (see the System Requirements and Download section). In-code remarks placed after two forward slashes are ignored by the program (e.g., //major losses).

Code in the MartiansV2 syntax consists of three different sections: definitions, structures, and experiment.

\section{Definitions}

This section serves two purposes. On the one hand, the definitions allow for simple forward-string-replacement declarations. The first definition in the example shows how, instead of reusing certain RGB values, one can define content of the string "BLUE" as "0.0.255". The second and third definitions make referring to specific stimuli easier.

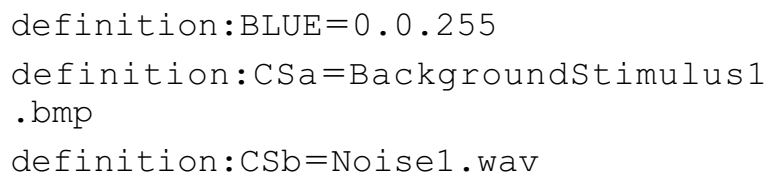

The second purpose served here concerns changing settings that apply to the entire experiment. Certain definitions are reserved and are interpreted before experiment execution, in order to adjust certain general computer settings. In this case, these are, respectively, screen resolution (width and height), number of colors, and full-screen mode (now switched off).

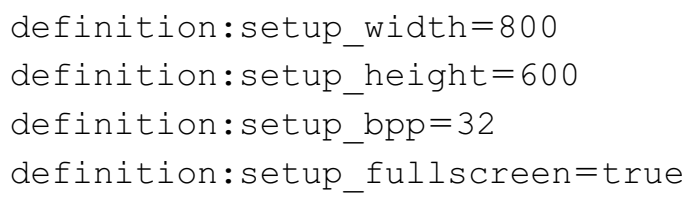

\section{Structures}

MartiansV2 has four types of structures: flashes, attacks (and invasions), trials, and blocks. Each structure has its own set of properties with different possible values. As can be expected, some structures function as building blocks for other structures. For example, blocks require attacks (and invasions) and trials, whereas flashes can be used in both attacks (invasions) and trials. An example from each type of structure will be discussed.

Flashes. In the following example code, flashes are created and named "F1". Flash properties include the frequency and color of the flashes. "F1" flashes can now be a part of trials and invasions.

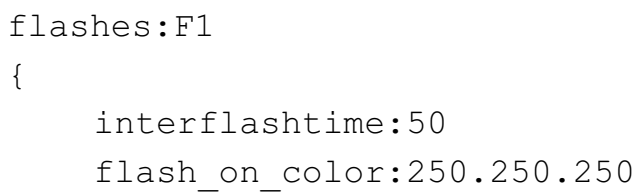

Attacks and invasions. An attack is created and named "Normal_attack". Five properties of attacks with their respective values are declared. First, the speed by which the Martians arrive on the screen is set to $4 / \mathrm{sec}$ (one Martian each $250 \mathrm{msec}$ ). The bitmaps for Martians and explosions are defined. Finally, the attack is indicated not to be an invasion.

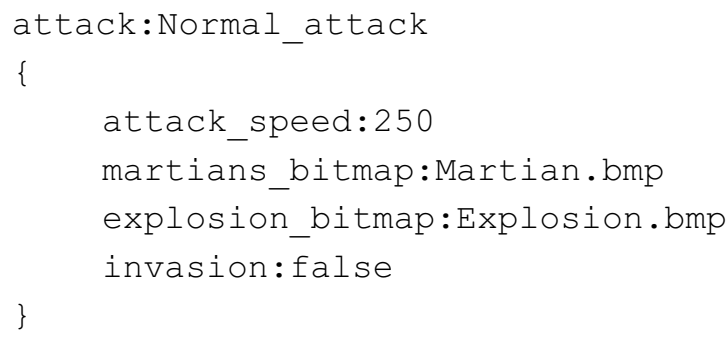

The invasion is created and named "Normal_invasion". Since it partly uses the same values for properties as the previously defined "Normal_attack", the latter can be used as a template. This way, "Normal_invasion" starts off with the same property settings as "Normal_attack", except for properties explicitly added or modified. A similar rationale will be used for blocks and trials. The invasion property is set to "yes". Since the Martians' attack speed is 
faster during invasions than during attacks, it is reset to 10 Martians/sec. As invasion flashes, "F1" is set; as invasion looping sound, "invade.wav" is set. Also, the invasion is set to be unavoidable and unconditionally $5 \mathrm{sec}$ long. During the invasion, the Martians cannot be killed. Therefore, the Martians are set to be "immune" for the laser.

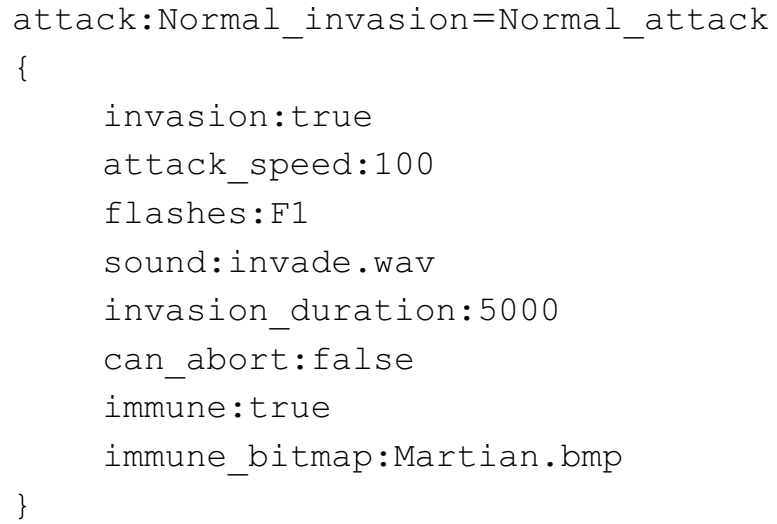

Trials. Trials are structures with, among setting properties, the event property. Events are time-based declarations, always preceded by a dead time of the number of milliseconds stored in the "initial_delay" property. There should be no delay before events occur after trial onset. Therefore, the "initial_delay" is set to 0 for all trials in this experiment by creating a trial named "Template_ Trial", which will be used as an outline for the other experimental trials. The "save_keystrokes" command allows for storing all moments during which a barpress is registered. This rationale of using templates will be especially useful while creating blocks later on.

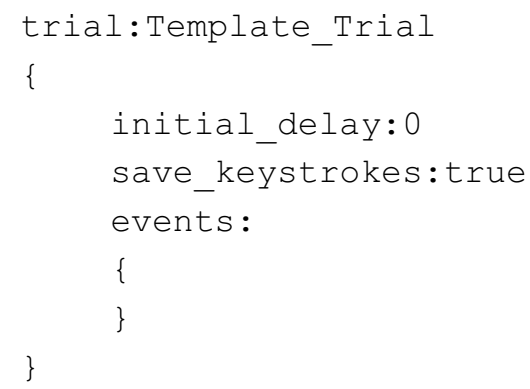

As an example, the reinforced trial with stimulus CSa is discussed. A trial named "CSa_Reinforced" is created and set equal to the "Template_Trial". The following events are added: The visual stimulus "CSa" (see the Definitions section) is displayed on screen between 1.5 and $3 \mathrm{sec}$ after trial onset (since the "initial_delay" is set to 0 ). The presentation of the antilaser shield (US), consisting of the "F1" flashes and "shield.wav" sound, follows the reinforced CS immediately and remains on screen for $500 \mathrm{msec}$ ( 3 to $3.5 \mathrm{sec}$ ). Simultaneously with the US, "Normal_invasion" is activated. During its activation - in this case, USpresentation - the invasion will be triggered conditional on a barpress. Calculating suppression ratios (SRs) requires two intervals of barpress counts, one during CS presentations ("B") and one during a time interval equal in length before CS presentation ("A"). The "start_measuring()" and "stop_measuring()" commands will be discussed further in the Output section. We would also like to indicate that there is an option for administering electrical shocks (for more information, please see the Web site).

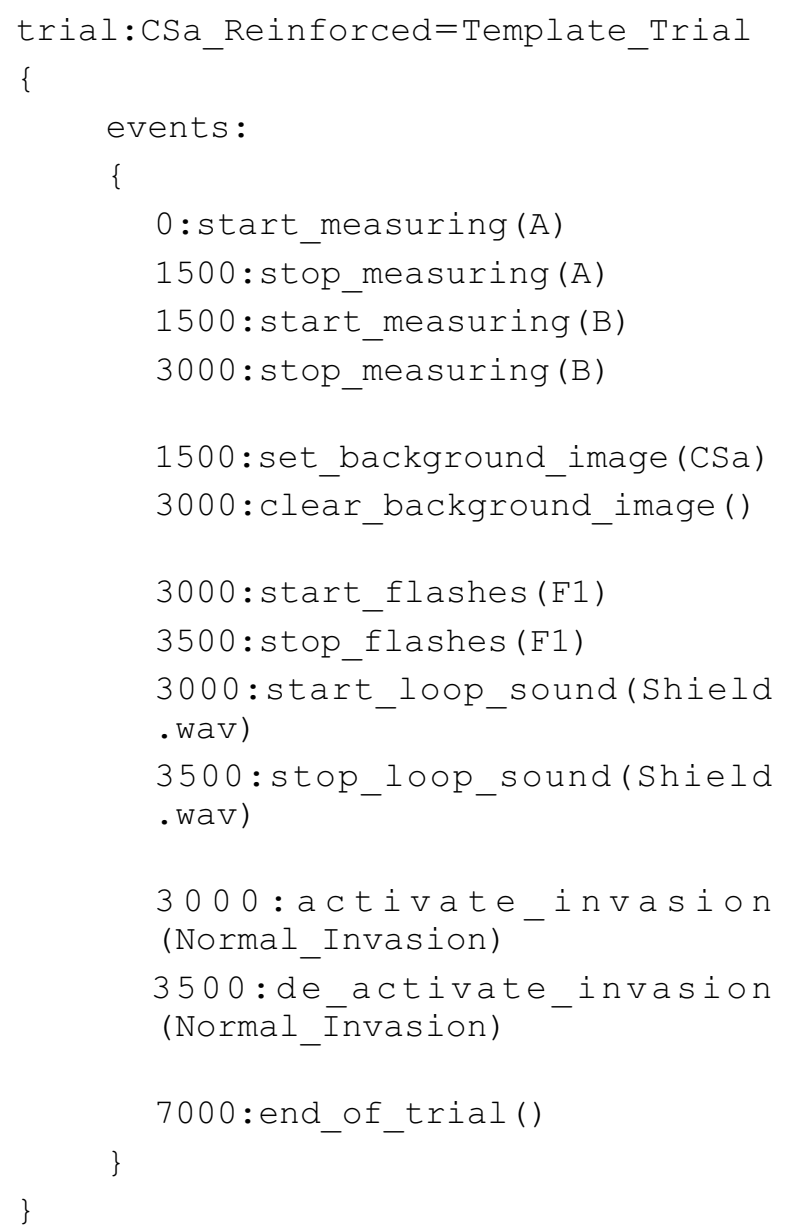

Blocks. Blocks are hierarchically the highest structures. They have their own properties and function as configurations of attacks, invasions, and trials. Since they have many properties that will not change very often during an experiment, creating a template will prove its worth later on. ${ }^{2}$ The block "Template_Block" is created. The Martians will appear on the screen in 10 columns and 7 rows, with both horizontally and vertically 20 pixels in between them. The drifting and the smooth progression of new columns or rows of Martians is activated at an appropriate speed. The trial order within each block is set to be semishuffled - that is, randomized, with the restriction that no more than two trials of the same type be presented consecutively. The background color is set. Finally, the attack and invasion for the block are chosen.

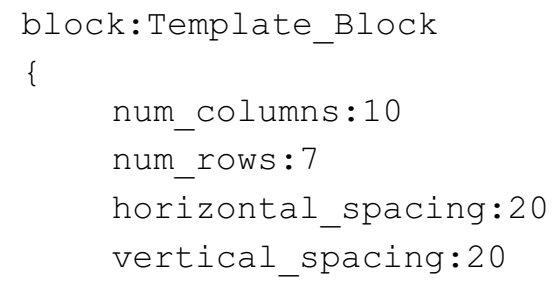


drifting:true

drift_factor: 5

trial_order:semi-shuffled

background_color:BLUE

attack_formation:Normal_attack

invasion:Normal_invasion

\}

An acquisition block is created as equal to the "Template_Block" and named "Acquisition". In fact, compared with "Template_Block", only the trials of the block have to be added for it to be usable in the experiment section. The two trial types "CSa_Reinforced" and "CSb_Unreinforced" are both presented three times.

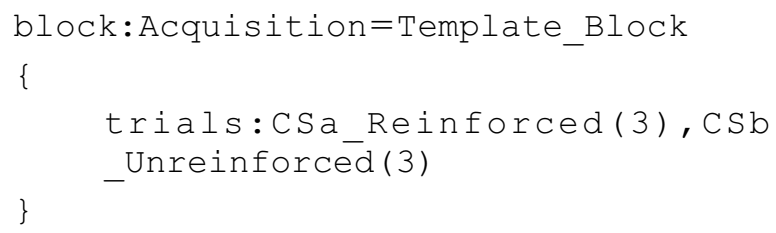

\section{Experiment}

The experiment section basically represents the time line of the experiment. It contains from top to bottom, in chronological order, what stimuli, instructions, and feedback will be displayed on screen. The first command allows the ITI to be changed by adjusting the mean and deviation from the mean time (in milliseconds). The second command sets the default background color. Note that it uses the definition of "BLUE" specified before. The background color specified here is applied, but it can be overruled temporarily at the block and event levels.

$$
\begin{aligned}
& \text { set_itt }(7500,2500) \\
& \text { set_background_color (BLUE) }
\end{aligned}
$$

The following commands do exactly what their names imply. Once the subject's ID is entered, the screen is restored to the blue background and the first set of instructions is displayed. Next, the program waits for a mouse click to continue.

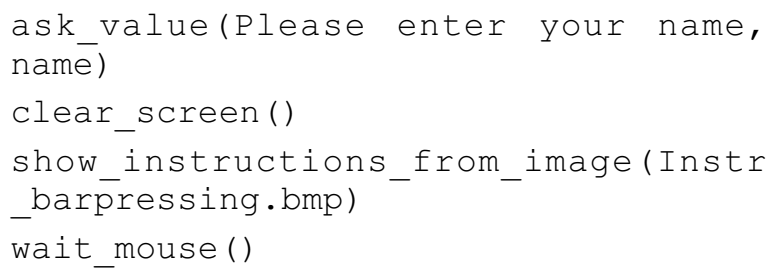

This gives the subject time to read the instructions, and allows the experimenter to give further instructions verbally. When the mouse is clicked, the instructions give way to the blue screen and, after $2 \mathrm{sec}$, the first Martians appear in the barpressing pretraining.

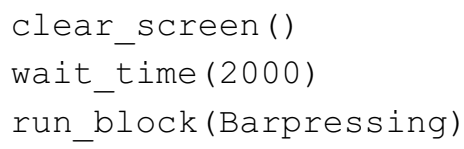

Immediately after the barpressing phase, subjects receive feedback on their percentage of hits. When they continue the experiment by clicking the mouse, their score of hits and misses is reset.

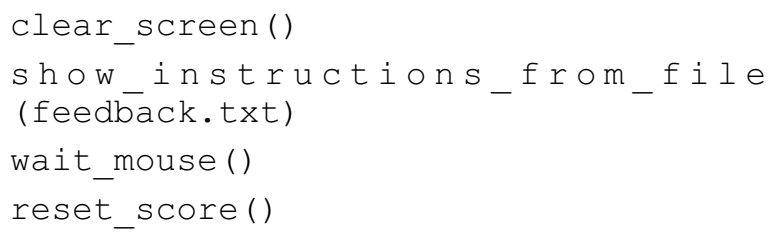

The following experimental block, the US-only phase, is introduced and executed, after which feedback is provided and the final instructions are presented.

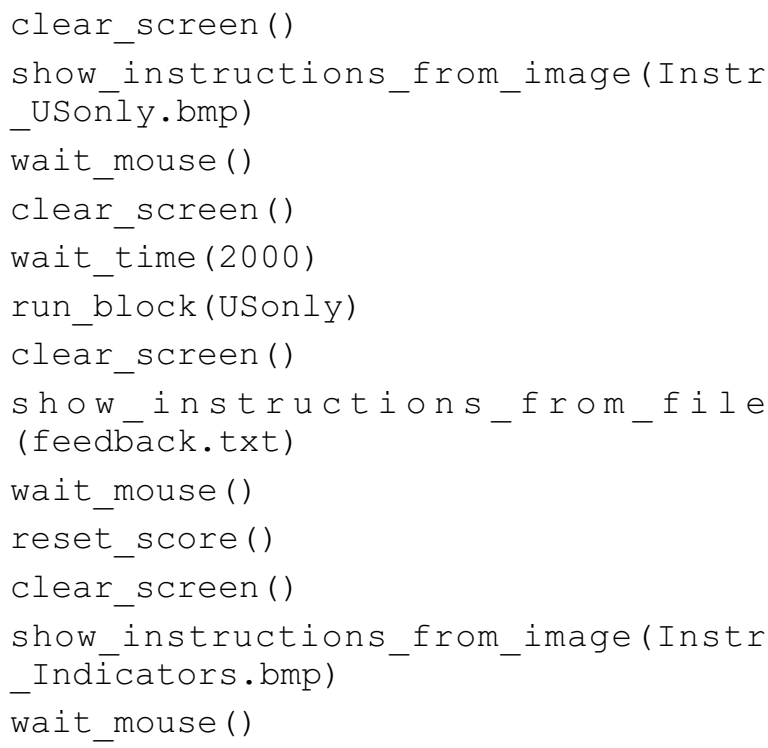

When pretraining is finished, the actual experiment can begin. The test phase follows the acquisition phase without delay. The transition between blocks is smooththat is, the attacks are not interrupted when a new block starts.

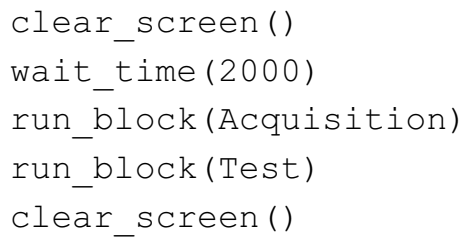

Again, feedback is provided. After displaying a "thankyou note" to the subject, MartiansV2 closes.

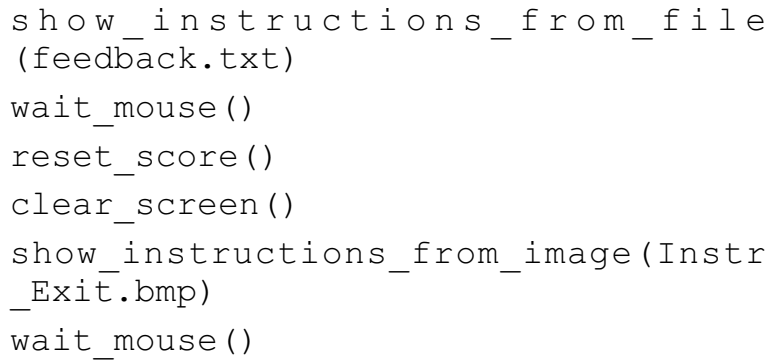




\section{RUNNING AN EXPERIMENT}

Make sure all the files, including the bitmaps, sounds, code (and dll's and .exe for Windows users), are in the same folder. Windows users can simply double-click the MartiansV2.exe and choose the code they want to use; Mac users can start MartiansV2 directly from the Textwrangler scroll menu.

\section{OUTPUT}

Aiming for simplicity and flexibility in use, we decided to choose Microsoft Excel's pivot table function as the intended tool for manipulating MartiansV2 output. When an experiment is finished, all output is written to a ttxt file in the results folder. If that file is opened with Excel, the following column headers can become visible (depending on the experiment): "filename", "name", "date", "Block Name", "Block_Num", "Block_Hits", "Block_Misses", "Trial_Name", "Trial_Num", "Attack", "Invasion_ running", "Event", "Trial_Time", "Block_Time", "Barpresses", "a", and "b". The latter two columns contain the barpress counts the experimenter requested via the "start_ measuring()" and "stop_measuring()" events. Each time MartiansV2 encounters a new argument for these commands, a new output column is created. Thus, the "a" and "b" columns contain the dependent variables, the counts. In order to obtain suppression ratios, in this particular case, all one needs to do is create a new column to compute the $\mathrm{SR}=\mathrm{b} /(\mathrm{b}+\mathrm{a})$ ratio. The "Barpresses" column contains all moments during which MartiansV2 encountered a barpress. This function can be switched on and off using the trial-level command "save_keystrokes". All columns contain exactly what their names imply. After creating a pivot table and dragging the SRs into the Data field, one can mold the data by removing and adding dependent variables (e.g., "Block_Name" and "Trial_Name") to the different fields until the data is ready for use in any statistical software package. We emphasize that the dependent variables will be continuously updated and stored by MartiansV2 as long as the "stop_measuring()" command is not encountered. Therefore, it is usually best to include only the "end_of_trial()" events, which is easily done in the pivot table. A short introduction on the MartiansV2 output, and how to import, explore, and manage the data, is located at the Web site under the "Output" tab.

\section{SYSTEM REQUIREMENTS AND DOWNLOAD}

MartiansV2 was designed to be cross-platform; the code is editable and runnable in Windows XP and 2000 as well as in Mac OS X. It can, in principle, be validly used on any modern computer capable of running one of the aforementioned operating systems.

MartiansV2 can be downloaded from the Web page of the KULeuven Centre for the Psychology of Learning and Experimental Psychopathology (http://ppw.kuleuven.be/
leerpsy/MartiansV2/). Under the tab "Download" are the different versions for machines running on Windows XP and 2000 and Mac OS X, as well as several examples of experiments, including the one presented here, and instructions for use and download.

\section{IMPLEMENTATION}

Disclaimer, Bug Reports, and Feature Requests

MartiansV2 is a work in progress. New feature requests or bug reports can be submitted at the following Web site: http://ppw.kuleuven.be/leerpsy/mantis/login_page .php. All currently implemented features have been tested thoroughly. However, the authors reserve the right to decline feature requests and disclaim any responsibility for errors.

\section{AUTHOR NOTE}

This research was supported by GOA funding (GOA 3H051018). Correspondence concerning this article should be addressed to M. Franssen, Department of Psychology, University of Leuven, Tiensestraat 102, B-3000 Leuven, Belgium (e-mail: mathijs.franssen@psy.kuleuven.be).

\section{REFERENCES}

Arcediano, F., Matute, H., \& Miller, R. R. (1997). Blocking of Pavlovian conditioning in humans. Learning \& Motivation, 28, 188-199. doi:10.1006/lmot.1996.0957

Arcediano, F., Ortega, N., \& Matute, H. (1996). A behavioural preparation for the study of human Pavlovian conditioning. Quarterly Journal of Experimental Psychology, 49B, 270-283.

Baeyens, F., \& Clarysse, J. (1998). Martians for Windows 95 [Computer program]. Leuven: University of Leuven.

Baeyens, F., Clarysse, J., Franssen, M., \& Hermans, D. (2008). MartiansV2 [Computer program]. Leuven: University of Leuven.

Baeyens, F., Vansteenwegen, D., Beckers, T., Hermans, D., KerkHOF, I., \& DE Ceulaer, A. (2005). Extinction and renewal of Pavlovian modulation in human sequential feature positive discrimination learning. Learning \& Memory, 12, 178-192. doi:10.1101/1m.89905

Baeyens, F., Vansteenwegen, D., Hermans, D., Vervliet, B., \& EELEN, P. (2001). Sequential and simultaneous feature positive discriminations: Occasion setting and configural learning in human Pavlovian conditioning. Journal of Experimental Psychology: Animal Behavior Processes, 27, 279-295. doi:10.1016/S0023 $-9690(03) 00058-4$

Baeyens, F., Vervliet, B., Vansteenwegen, D., Beckers, T., Hermans, D., \& Eelen, P. (2004). Simultaneous and sequential feature negative discriminations: Elemental learning and occasion setting in human Pavlovian conditioning. Learning \& Motivation, 35, 136-166. doi:10.1016/S0023-9690(03)00058-4

Beckers, T., De Houwer, J., Pineño, O., \& Miller, R. R. (2005). Outcome additivity and outcome maximality influence cue competition in human causal learning. Journal of Experimental Psychology: Learning, Memory, \& Cognition, 31, 238-249. doi:10.1037/0278 $-7393.31 .2 .238$

Fonteyne, R., \& Baeyens, F. (2009). Extinction and ABA, ABC, and $A A B$ renewal of Pavlovian modulation in human sequential feature positive discrimination learning. Manuscript in preparation.

Franssen, M., Gillard, E., Dirikx, T., van Vooren, P. R., \& BaeYENS, F. (2009). Reinstatement of Pavlovian modulation. Manuscript in preparation.

Havermans, R. C., Keuker, J., Lataster, T., \& Jansen, A. (2005). Contextual control of extinguished conditioned performance in humans. Learning \& Motivation, 36, 1-19. doi:10.1016/j.1mot.2004.09.002

Joos, E., Vansteenwegen, D., \& Hermans, D. (2008, September). Consolidation of the CS-US association through mental rehearsal in two different paradigms. Poster presented at the 38th Annual Congress 
of the European Association for Behavioural and Cognitive Therapies (EABCT), Helsinki, Finland.

LipP, O. V., \& Dal Santo, L. A. (2002). Cue competition between elementary trained stimuli: US miscuing, interference, and US omission. Learning \& Motivation, 33, 327-346. doi:10.1016/S0023 $-9690(02) 00001-2$

Matute, H., \& PineÑo, O. (1998). Stimulus competition in the absence of compound conditioning. Animal Learning \& Behavior, 26, 3-14.

Matute, H., Vadillo, M. A., \& Bárcena, R. (2007). Web-based experiment control software for research and teaching on human learning. Behavior Research Methods, 39, 689-693.

Meulders, A., Vervliet, B., Vansteenwegen, D., Hermans, D., \& BAEYENS, F. (2009). A new tool for assessing context conditioning induced by US unpredictability in humans: The Martians task restyled. Manuscript submitted for publication.

\section{NOTE}

1. A "text" editor is not a word processor such as MS Word. A text file is a nonstyled document: No formatting of layout is included, only text and line breaks. Although the recommended file extension is .mrs2, the actual content is plain text. One can safely create .txt files and edit these; the disadvantage is that the icon of the experiment file will appear as a text file.

2. Note that one could create two block templates for two different experimental contexts. This way, attacks, invasions, backgrounds, and so forth, can be changed easily, using the different templates.

(Manuscript received May 18, 2009;

revision accepted for publication July 29, 2009.) 